Lingüística

Vol. 36-2, diciembre 2020: 51-69

ISSN 2079-312X en línea

DOI: $10.5935 / 2079-312 X .20200014$

\title{
FONOLOGÍA DEL TUCANO OCCIDENTAL EN UN VOCABULARIO MISIONERO DEL ESPACIO GEOGRÁFICO ECUATO-COLOMBIANO (SIGLO XVIII) ${ }^{1}$
}

\author{
A FONOLOGIA DO TUCANO OCIDENTAL EM UM VOCABULÁRIO MISSIONÁRIO \\ DO ESPAÇO GEOGRÁFICO ECUATO-COLOMBIANO \\ (SÉCULO XVIII)
}

\begin{abstract}
PHONOLOGY OF WESTERN TUCANO IN A MISSIONARY VOCABULARY OF THE ECUATO-COLOMBIAN GEOGRAPHICAL DOMAIN

(18TH CENTURY)
\end{abstract}

\author{
Micaela Carrera de la Red \\ Universidad de Valladolid \\ micaela@fyl.uva.es \\ 0000-0002-8876-844
}

\begin{abstract}
Resumen
En este artículo se analiza la fonología de la lengua amazónica descrita en un Vocabulario fruto de la acción misionera por parte de los Padres Franciscanos del convento de Quito en las cuencas de los ríos Putumayo y Caquetá a lo largo del siglo XVIII. Se parte del carácter multiétnico y multilingüe de la población indígena de la actual área ecuato-colombiana. Se plantea la hipótesis de que el Vocabulario recoge una "lengua general" que facilitaría la comunicación entre diferentes naciones de indios. Los rasgos se extraen de la grafémica de dos manuscritos del Vocabulario puesta en conexión con las funciones fonológicas que se asignan a variantes gráficas y grafemas en estudios sobre lenguas amazónicas. Entre las conclusiones destaca que cada uno de los dos manuscritos manejados del Vocabulario refleja una proporción diferente de rasgos fonológicos. En el llamado Ms-RAH es mayor la presencia de rasgos fonológicos de la lengua siona, mientras que en el llamado Ms-BNC prevalecen los rasgos procedentes de la lengua koreguaje.
\end{abstract}

\footnotetext{
${ }^{1}$ Esta investigación forma parte del Proyecto I+D+i Análisis histórico del discurso en el español de Colombia (Ref. 2012-31205). En este trabajo se ofrece una versión reformada y sintetizada de dos ponencias orales, en las cuales se esbozaban aspectos, muchos de los cuales se concretan aquí, sobre la filiación de los manuscritos y sobre el léxico (Carrera de la Red y Zamora 2014) y sobre la grafémica y los correlatos fonológicos (Carrera de la Red 2015).
} 
Palabras clave: tucano occidental; grafémica; fonología contrastiva; lingüística misionera; región ecuato-colombiana.

\title{
Resumo
}

Este artigo analisa a fonologia da língua amazônica descrita em um vocabulário como resultado da ação missionária dos padres franciscanos do convento de Quito nas bacias dos rios Putumayo e Caquetá ao longo do século XVIII. Partese do caráter multiétnico e multilíngue da população indígena da atual área equatorial-colombiana. Supõe-se que o vocabulário contém uma "linguagem geral" que facilitaria a comunicação entre as diferentes nações indígenas. Os traços são extraídos do grafismo de dois manuscritos do Vocabulário colocados em conexão com as funções fonológicas atribuídas a variantes gráficas e grafemas nos estudos das línguas amazônicas. Dentre as conclusões, destacase que cada um dos dois manuscritos gerenciados do Vocabulário reflete uma proporção diferente dos traços fonológicos. No denominado Ms-RAH a presença de traços fonológicos da língua siona é maior, enquanto no denominado MsBNC prevalecem os traços oriundos da língua koreguaje

Palavras-chave: tucano ocidental; grafêmica; fonologia contrastiva; linguística missionária; região equato-colombiana.

\begin{abstract}
This article analyzes the phonology of the Amazonian language described in a Vocabulary resulting from the missionary action of the Franciscan Fathers from the convent of Quito in the basins of the Putumayo and Caquetá rivers throughout the 18th century. It is based on the multiethnic and multilingual character of the indigenous population of the current Equatorial-Colombian area. It is hypothesized that the Vocabulary includes a "general language" that would facilitate communication between different nations of Indians. The traits are extracted from the graphemic analysis of two manuscripts of the Vocabulary put in connection with the phonological functions that are assigned to graphic variants and graphemes in studies on Amazonian languages. Among the conclusions, it is highlighted that each of the two manuscripts of the Vocabulary reflects a different proportion of phonological features. In the socalled $M s-R A H$, the presence of phonological features from the Siona language is greater, while in the so-called Ms-BNC the traits coming from the koreguaje language prevail.
\end{abstract}

Key words: Western Tucano; graphemics; contrastive phonology; missionary linguistics; Equatorial-Colombian region.

Recibido: $14 / 01 / 2019$

Aceptado: 24/04/2019 


\section{Corpus: difusión y dependencia entre los manuscritos del Vocabulario}

Debemos al Padre Fray Jesús María Larrea uno de los escasos testimonios referidos a esa compleja realidad etnolingüística, que es el tucano occidental de la época colonial, a quien pasamos a considerar desde aquí autor de esta obra misionera (Jiménez de la Espada 1898-1899, 1904, v. Mantilla 1986) ${ }^{2}$. Se trata de diversos textos manuscritos fechados a lo largo del siglo XVIII, fruto de la actividad misionera en la Amazonía occidental realizada desde el convento franciscano de San Diego de Quito. El núcleo central de estos manuscritos lo constituye un Vocabulario (de alrededor de 1625 entradas léxicas) de una lengua perteneciente a la familia tucana, con la traducción en castellano, y está acompañado de otros textos que cambian según los manuscritos: un listado de verbos, unas apuntaciones gramaticales sobre la lengua amazónica o la doctrina en la misma lengua.

Para avanzar en un mejor conocimiento de estas fuentes, es necesario señalar algunos indicadores de dependencia entre los manuscritos conservados y, a partir de ese análisis, retomar la idea esbozada con anterioridad sobre la autoría de este Vocabulario.

El primer manuscrito que se analiza en esta ocasión es el más antiguo de esta obra misionera, según la fecha situada al final del texto (Anno Domini, 1751); lleva el título de Vocabulario de la lengua de los indios, que poblan [sic] los ríos de Putumayo, y Caqueta, hecho á solicitudes del. Colegio de Missiones de San Diego de Quito. Este manuscrito llegó a España y forma parte de los fondos de la Real Academia de la Historia de Madrid (de ahí que, a partir de ahora, lo llamemos Ms-RAH) ${ }^{3}$. Con cierta probabilidad fue modelo de otros manuscritos que hoy se encuentran en distintas bibliotecas parisinas ${ }^{4}$. Lo editó el erudito Marcos Jiménez de la Espada (1898-1899, 1904), este último publicación póstuma de manera independiente en la misma editorial ${ }^{5}$.

${ }^{2}$ El padre Fray Fernando de Jesús Larrea, franciscano quitense, fue ordenado sacerdote en 1725 en el convento de la Recolección de San Diego de Quito. De este padre franciscano se refiere su tarea como predicador de misiones populares desde el año 1732, primero en Quito, con ocasión de las fiestas en honor de la Virgen del Quinché, y en 1739 en Popayán para revitalizar el convento de misiones que allí había, hasta transformarlo en el famoso Colegio de Propaganda Fide de la ciudad; en 1742 predicó en misiones populares en el Valle del Cauca, Cundinamarca, Boyacá, Santander, Tolima y Cali, ciudad en la que fundó en 1757 el Colegio de Misiones de San Joaquín de Cali, que aún pervive como convento de San Francisco (Zawadzky 1947).

${ }^{3}$ Los repertorios sobre lenguas amerindias sitúan el Ms-RAH como anónimo (Tovar 1961: 212, Tovar y Larrucea de Tovar 1984: 230), lo mismo que el Ms-BNC y el Ms-BPR, este último situado entre los anónimos (Tovar 1961: 214, Tovar y Larrucea de Tovar 1984: 232 y Larrucea de Tovar 1986: 213-229).

${ }^{4}$ Todos ellos copias de fechas más tardías al Ms-RAH (Madrid): en la Médiathèque du musée du quai Branly, y en la Biblioteque du Musée de I'Homme. Son los mismos que Landaburu (1996) señala como citados por Paul Rivet en su archivo de manuscritos de lenguas indígenas amazónicas (Msl00, año 1791, con el título Lenguas del Putumayo, Caquetá, a solicitud del Colegio apostólico de San Diego de Quito, Ms124 y Ms229, ambos copias de fechas muy tardías, 1920).

${ }^{5}$ Está mal catalogado en la Biblioteca de la Universidad de Salamanca en lo referente a la fecha: figura como de 1964. 
El segundo manuscrito que se toma en cuenta no tiene fecha; se halló en el Colegio Propaganda Fide de Popayán y figura como "Raro Manuscrito 122" (Ortega Ricaurte 1997: 1146) en el índice de lenguas indígenas de la Biblioteca Nacional de Colombia (Bogotá) (a partir de ahora Ms-BNC). Con el título de Bocabulario de la lengua que usan los indios de estas misiones. Ceona, fue publicado por el historiador y etnógrafo colombiano Sergio Elías Ortiz (1942: 137-199 $)^{6}$. Al final del $M s-R A H$, se sitúa un compendio gramatical. En cambio, en el Ms-BNC, antes del Bocabulario, encontramos una selección de verbos que se repiten en el glosario; detrás del vocabulario aparecen dieciocho páginas más con la Doctrina cristiana y, como colofón, otro listado de Vocablos para hacer más comprensible la lengua de estas misiones (Ortega Ricaurte 1997: $1146)^{7}$.

\section{Lengua general, lengua de la familia tucana y lengua siona}

El propio Jiménez de la Espada, en su edición del Vocabulario, ofrece una reflexión sobre el término "general" de la lengua a la que se supone que pertenece:

La calificación de general que el autor asigna al idioma de su vocabulario revela, en mi juicio, que no era (o es) peculiar de nación determinada, muy densa o difundida o dominadora, y que el calificativo equivale al de franco, como el que se aplica al que emplean para facilidades de comunicación y comercio los ribereños de nuestro Mediterráneo, y usaban los indios yuncas litorales del Perú. Y si este mi parecer es inadmisible, tras él viene la sospecha de que el léxico de los franciscanos quitenses pueda ser el de un lenguaje no propio y exclusivo de los indios del Yapurá y Putumayo, pero mixto de otros cercanos y afines y compuesto y ordenado para la mayor comodidad y expedición de su negocio evangélico (Jiménez de la Espada 1898-1899, T. II, 5: 200).

Lo interesante es que el Ms-RAH contiene un sistema "mixto" de lenguas. En esto Jiménez de la Espada muestra dependencia de algunas fuentes históricas, ya que secunda la afirmación del misionero jesuita Gilij (1780) de que la lengua del Vocabulario corresponde a la familia denominada maipure (Jiménez de la Espada 1898-1899, T. II, 5: 200). Vinculado con la familia arawak, el maipure era un grupo que por su nivel de extensión, junto con el caribe-arawak, tenía posibilidades de llegar a convertirse en "lengua general", según cita de Gilij (1780) recogida por González Mora (2004: 33): "[el

6 En el momento actual, junto con los demás manuscritos de la Biblioteca Nacional de Colombia, está siendo transcrito por etnógrafos colombianos del Instituto Colombiano de Antropología e Historia (Gómez y Torres 2012-2013).

7 En nuestro análisis no tomamos en cuenta un tercer manuscrito del mismo Vocabulario perteneciente a los "Fondos americanistas", "Colección Mutis", de la Real Biblioteca del Palacio Real (Madrid) (Ms-BPR). Este manuscrito, del que consta que se copió en julio de 1788 y que es identificado como Vocabulario de la lengua siona (Moreno Gallego 2009: 105-140), utiliza el mismo tipo de orden preciso: $A$ ante $B, A$ ante $C$, etc., que en el Ms-BNC, lo que puede apuntar a una relación de dependencia entre ambos manuscritos. 
maipure] lo entienden todos en el gran río y se podría hacer común si se quisiera [...] por lo tanto, de persistir el obstáculo de tantas lenguas... ésta [la lengua maipure] sería bastante a propósito para hacer de ella una lengua general" 8 .

Había que esperar a que el foco se dirigiera hacia otras tribus de las áreas occidentales, como son los pobladores de las cuencas de los ríos IçaPutumayo, su afluente el río Napo y el río Yapurá-Caquetá.

A mediados del siglo XX, en el estudio previo a la edición del Ms-BNC, el historiador Sergio Elías Ortiz (1942: 138) sostiene también que la lengua fijada por escrito es una "lengua general" pero afirma que coincide con la lengua ceona o siona, idioma que pertenece al grupo occidental de la familia lingüística tucana, junto con el amaguaje, el tama, el koreguaje, el makaguaje, el ikaguate, el pioje $\mathrm{y}$, seguramente, el koto, agregado posteriormente al grupo de este sector por Loukotka $(1935)^{9}$. Unas décadas después, Tovar (1961: 212) vincula, de nuevo, la lengua del Ms-RAH al tucano, de igual manera que Tovar y Larrucea de Tovar (1984: 230), aunque en esta ocasión logran una mayor precisión al situarlo en el tucano occidental.

Pero en ningún caso precisan de qué lengua se trata dentro del subgrupo occidental.

\section{Metodología del análisis}

La propuesta que se hace desde aquí se basa en un estudio sistemático de los rasgos lingüísticos que guardan conexión con la lengua siona, considerada la base de este Vocabulario, frente a aquellos rasgos que pertenecen a otras lenguas tucanas occidentales (koreguaje, secoya), e incluso con los de otras lenguas de la Amazonía, septentrional y meridional. En esta ocasión, los ejemplos se extraen únicamente a partir del Vocabulario ${ }^{10}$.

Si se tiene en cuenta que las lenguas amazónicas son ágrafas, el estudio de los grafemas y variantes gráficas, con los correlatos fonéticos y fonológicos que les corresponden, es el ámbito de estudio más idóneo para investigar esta hipótesis, ya que las estrategias adoptadas en la confección de la escritura de lenguas tan distintas a las europeas en fonética y en fonología tienen que ser muy precisas.

${ }^{8}$ Es lícito pensar que los parámetros etnográficos y sociolingüísticos que Sorensen 1972: 7893) y Jackson (1974: 50-64, 1983, 2001: 373-396) utilizan para explicar el multilingüismo de las tribus indígenas de la Amazonía en el siglo XX pueden allanar el camino para comprender el fenómeno del multilingüismo de estos pueblos en otros momentos históricos. El objeto del estudio de Sorensen 1972: 88) son los indios de la familia tucana pobladores del área noroccidental del Amazonas central, en torno al río Vaupés y sus afluentes.

${ }^{9}$ Según información contenida en el Portal de las Lenguas Indígenas de Colombia del Instituto Caro y Cuervo (Bogotá), en la parte correspondiente hoy día a Colombia, la familia tucano occidental está distribuida por los actuales Departamentos colombianos de Putumayo y de Caquetá, mientras que las familias uitoto y bora están distribuidas por el Departamento del Amazonas.

${ }^{10}$ La Gramática se ha tomado en consideración en otro trabajo enfocado, esta vez, sobre los "clasificadores", recurso de formación nominal propio de la estructura gramatical de las lenguas amazónicas (Carrera de la Red 2016). 
La perspectiva más adecuada para la interpretación de las soluciones grafémicas adoptadas por los misioneros en estos textos es la comparativa; en primer lugar, se establece la comparación entre las variantes grafémicas de los dos manuscritos principales (por su proximidad al texto originario) del Vocabulario y los posibles correlatos que se les atribuyen desde la fonología y, en segundo lugar, entre esas variantes y la estructura fonológica de las distintas lenguas amazónicas en la actualidad ${ }^{11}$.

De forma principal, se toma en cuenta la fonología de las tres lenguas del subgrupo tucano occidental (siona, koreguaje y sekoya). La metodología de trabajo trata los datos extraídos de este Vocabulario quitense en comparación con aquellos proporcionados por los estudios de las lenguas amazónicas de esa área en épocas más actuales reducirá el impacto de las limitaciones que sobre las conclusiones a las que se pueda llegar se crean tanto por el factor diacrónico como por la distancia geográfica, etnográfica y cultural.

\section{Bosquejo del inventario grafémico del Vocabulario}

Al carecer estas lenguas de fijación gráfica, se trabaja con una ortografía de tipo fonémico $y$, en la búsqueda de regularización de los símbolos fonémicos, se eliminan las variaciones ortográficas sin prestar atención a las correspondencias con el sistema fonológico. Como excepción, cabe mencionar la breve alusión, en un manual sobre las lenguas indígenas sudamericanas (Fabre 1998), a las "variaciones ortográficas" que se observan en la lengua siona actual entre $\langle\mathrm{y}\rangle \mathrm{e}\langle\mathrm{j}\rangle$, 0 entre $\langle\mathrm{h}\rangle \mathrm{y}\langle\mathrm{g}\rangle$, así como a la variación ortográfica vigente en palabras que empiezan por $\langle$ c- $\rangle$, $\langle$ hua- $>$ y $<$ gua- $>$.

También son importantes las Notas sobre ortografías que constan en la presentación del volumen publicado por el Instituto Lingüístico de Verano (Summer Institute of Linguistics) sobre los sistemas fonológicos de idiomas de Colombia, entre ellos el koreguaje (Muller de Young et al. 1984: 63-79). Los autores de este volumen explican la ortografía que utilizan en sus ejemplos; donde ellos hablan de "ortografía" podría hablarse de grafémica, porque se trata de elegir entre $l a\langle h\rangle$, por su carácter fonológico de sorda en español, o el saltillo $\langle '\rangle$, como grafías más adecuadas para representar la oclusiva glotal $/ 2 /$, o de fijar el dígrafo <ch> para la africada alveopalatal /č/, así como la grafía $<$ c > para la oclusiva velar sorda /k/ o los dígrafos <sh $>$ y $<$ zh $>$ para las

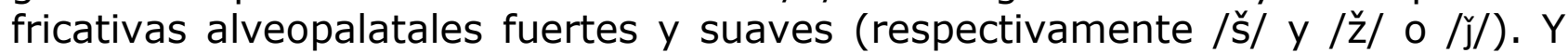
así con el resto de sonidos que difieren del español en las lenguas amazónicas.

En este recorrido 'ortográfico' puede encontrase un símil del proceso de selección del inventario de grafemas vocálicos y consonánticos, que pudo seguir el misionero franciscano en el siglo XVIII para la ardua y fructífera tarea de escrituración de la lengua amazónica que ayudara en la evangelización de

\footnotetext{
${ }^{11}$ En los últimos años se han producido avances significativos en la descripción de la fonología en las lenguas de la familia tucana: lenguas tatuyo y barasana (Gomez-Imbert 2000, 2003, 2004, 2007), lengua kubeo (Chacon 2012), lengua wanano (Stenzel 2007), lengua desano (Silva 2012), secoya del Putumayo (Vallejos 2013: 67-100), secoya del Aguarico (Ecuador) (Johnson y Peeke 1962, Johnson y Levinsohn 1990), entre otros. Existen distintos trabajos recientes de tipo comparativo y reconstructivo con aportaciones relevantes sobre el sistema consonántico del proto-tucano occidental (Skilton 2013, Chacon 2014: 275-332).
} 
estas etnias. Desde la grafémica, los conceptos de 'vocalismo' y 'consonantismo' no se entienden en el sentido de dos componentes de la fonología, sino en el sentido de la consideración de la existencia de grafemas vocálicos y grafemas consonánticos.

Son especialmente complejas las representaciones grafémicas vinculadas a la nasalización, así como los grafemas destinados a representar las consonantes oclusivas y fricativas de la serie alveopalatal y todo lo relacionado con los procesos llamados de laringalización (aspiración y glotalización) que recorren la fonología entera, segmental y suprasegmental, de las lenguas amazónicas y del prototucano.

\subsection{Representación grafémica de la nasalización}

La estructura básica de la lengua amazónica de estos manuscritos, en cuanto a raíces y también a morfemas, se corresponde con la descrita para el prototucano, de tipo monomoraica CV (Chacon 2014: 279), por lo que es poco probable que una sílaba termine en consonante, con la excepción de que la segunda vocal pueda sustituirse por un sonido glotal oclusivo CV(?) o fricativo $\mathrm{CV}(\mathrm{H})$. Existe también la posibilidad de una variante de la sílaba CVV, con la segunda vocal sorda del mismo timbre. La mayoría de las veces la secuencia vocálica es resultado de la confluencia de un radical y un sufijo: $C V+{ }_{(s u f)} V->$ CVV. En la mayoría de las ocasiones, en esos contextos morfonológicos el Ms$R A H$ y el Ms-BNC ponen las grafías de la secuencia vocálica. Desde el punto de vista comparativo de las lenguas amazónicas, es significativo que en siona dos vocales iguales seguidas se reducen fonéticamente a una sola (Wheeler 2000: 184), mientras que en koreguaje se mantiene la vocal doble como señal de su carácter largo (čoo [0:]) (Rodríguez González 2000: 203). Si nos centramos en los textos de los manuscritos Ms-RAH/Ms-BNC, nos encontramos con la presencia de ambas posibilidades, si bien los casos de mantenimiento de la secuencia vocálica son más frecuentes en el Ms-RAH que en el Ms-BNC:

(2)

Ms-RAH

Birá - Comenzar

Canimansicoo - La que sabe caminar

[-o morf. nom. fem. sing.]

Jaoó - Tender, arropar, ó abrigar

[-o morfema voz causativa]
Ms-BNC
Biraa - comensar, ó empesar
Canimansico - La que sabe caminar
Jao - Tender, arropar, ó abrigar

Cuando se mantienen las dos vocales, ambos manuscritos difieren en que el $M s-R A H$ señala la segunda vocal con un acento agudo $<{ }^{\prime}>$, mientras que el $M s-B N C$ sitúa la tilde de nasalidad $<\sim>$ también sobre la segunda vocal.

\section{(3)}

Ms-RAH

Carojaá - Por donde

Coó - La uña

\section{Ms-BNC \\ Carojaã \\ Coõ}

Los dos símbolos gráficos se relacionan con lo suprasegmental. El acento agudo es el diacrítico empleado en la descripción "grafo-fonémica" para indicar el tono alto dentro del acento tonal característico de estas lenguas (Cook y 
Criswell 1993: 2). Y la tilde sobre las vocales es índice de nasalidad, que en las lenguas amazónicas está vinculada a lo suprasegmental (Epps y Salanova 2013: 6). En las lenguas amazónicas la nasalidad no es una propiedad específica de consonantes o vocales, dado que su realización está siempre asociada con el morfema completo o la palabra (u ocasionalmente la sílaba) más que con segmentos particulares (Barnes 1999: 211, apud Epps y Salanova 2013: 4).

Otra representación de la nasalidad con alto grado de ocurrencia en los manuscritos del Vocabulario es el grafema alveolar posnuclear vocálico <-n> $y$, en mucha menor medida, el grafema bilabial <-m>. Desde la fonología, el contexto favorecedor de la inserción de esa consonante nasal en posición implosiva en tucano es aquel en el que la sílaba adyacente incluye una consonante oclusiva sorda (o fuerte, según Wheeler 2000: 182) en posición de ataque, creando una variante prenasalizada de la oclusiva.

\section{(4)}

Ms-RAH

Núncóa - Juntar, ó amontonar

/nũ'k ${ }^{h} o a /\left[n u ̃ n k^{h} w a\right]$

Encuepue - La nariz

/Pũ'hwebí/ (Wheeler 2000: 197)

Aunpiti - Pedazo de cabeza, ó pan de yuca

/ãũbiti/ [ãũbiti]

Aumpue - Buche, como quiera

/ãũbwe/ [ãũbwe]
$M s-B N C$

Nūncoa - juntar ó amontonar

Encuepue - la nariz

Aunpiti - pedasito de casabe, pan de yuca

Aunpue - buche que aquí dexa

Es decir, se inserta una nasal precisamente para bloquear la nasalización, así se frena su efecto sobre las otras sílabas sobre las que pudiera actuar la extensión de la nasalización, que afectaría a todos los segmentos sonoros que rodean al segmento nasal. Se trata de la llamada "armonía nasal", muy activa en las lenguas amazónicas (Epps y Salanova 2013: 5-6). Esto puede observarse en el verbo Cain - Dormir.

(5)

Ms-RAH

Cain - Dormir

\author{
Ms-BNC \\ /'kãĩ/ ['kñ̃̃i] 'dormir' \\ /'kãĩhã?jẽ/ ['khãĩ-hã ?nǣ] 'la siesta que va a dormir' \\ dormir-POT-PP.PRES. (='Aspecto potencial-Participio de Presente')
}

La pronunciación actual de este verbo en siona comporta nasalización de las dos vocales en un contexto de consonante precedente oclusiva velar sorda con aspiración $\left[\mathrm{k}^{\mathrm{h}}\right]$ en posición inicial radical. Por el contrario, en otras lenguas amazónicas, existen consonantes transparentes, que transmiten la nasalidad desde una vocal nasal a las vocales siguientes cuando las preceden. Estas son $/ \mathrm{w}, \mathrm{j}, \mathrm{h}^{\mathrm{w}}, \mathrm{h}, \mathrm{p} /$. En algunas de estas lenguas amazónicas, como la secoya, prácticamente todas las consonantes son transparentes a la nasalización (Vallejos 2013: 79-80). De hecho, no solo en siona sino en numerosas lenguas amazónicas se admiten similitudes perceptuales entre nasalización vocálica y otras prosodias, tales como la glotalización o la aspiración, fenómenos llamados de laringalización (Epps y Salanova 2013: 4-5). Esto podría explicar la solución grafémica adoptada en los dos manuscritos, el Ms-RAH y el Ms$B N C$, pero con mayor grado de coocurrencia en el segundo, para las secuencias 
vocálicas nasalizadas a base de marcar la separación de las vocales mediante el grafema $\langle\mathrm{h}\rangle$, cuyo correlato fonológico es glotal fricativo /h/.

(6)

Ms-RAH

Séhétoin - Caer otra vez

Payaá - Boltear

[-a morfema voz transitiva]

Choomay - No reir

[-o morfema voz causativa]

Choóco - La que ríe

Choóque - El que es risueño

Ciyoséé - Ahumado

[-é morfema pasado]

\author{
Ms-BNC \\ Seentoin \\ Paiaha \\ Chohomay - no reir \\ Chohoco - risueña \\ Chohoque - risueño \\ Ciyosehe - ahumado
}

Una última observación sobre la distribución de dos grafemas nasales en ambos manuscritos es que el grafema consonántico elegido en el Ms-RAH es el nasal palatal $<\tilde{n}>$, mientras que en el $M s-B N C$ es el grafema nasal alveolar $<\mathrm{n}\rangle$. La solución con mayor nivel de ocurrencia en el Ms-RAH se aproxima a la lengua koreguaje, ya que en el subgrupo tucano occidental, en un entorno de vocal nasal la consonante nasal sonora palatal $/ \mathrm{h} /$ tiene valor fonológico solo en la lengua koreguaje, mientras que en siona, secoya y orejón es una variante fonética de /y/ (Wheeler 1992: 22).

\section{(7)}

Ms-RAH

Ayiñé - Blando

Coañó - Ave nocturna

Ceñojañé - Color amarillo

\author{
MsBNC \\ Ahinẽ - blando \\ Coanã - Vna Ave nocturna \\ Cenõjanẽ - Color amarillo
}

Una de las conclusiones que puede extraerse de la revisión de esta parte del sistema grafémico es la confirmación de la indudable vinculación existente entre nasalización y laringalización (aspiración o glotalización) en la historia de las lenguas amazónicas.

\subsection{Grafema $<f>$}

Al revisar el Vocabulario vemos que son solo dos las entradas que aparecen debajo de la letra " $F$ ", junto con otras entradas, muy escasas en número, que lo llevan en posición medial:

(8)

Ms-RAH

Fio - silvar

Fioseé - silvido

Juéfué - Rebentar

Chafa - Regar agua

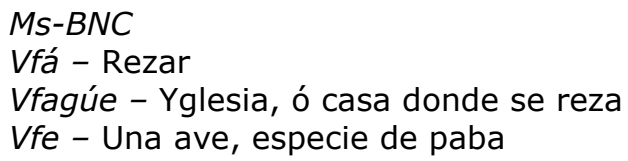

Ms-BNC

Vfá - Rezar

Vfagúe - Yglesia, ó casa donde se reza

Vfe - Una ave, especie de paba

Llevan este grafema palabras no pertenecientes al tucano occidental, si bien en prototucano se habla de la posibilidad de una fase intermedia / $\phi /$ en el proceso de laringalización de $* p>h$ en algunas variedades tucanas próximas a la lengua siona (Gomez-Imbert 2011). 
Pero la realidad es que hoy día, el sistema consonántico de las lenguas tucanas occidentales, tanto siona como koreguaje, no posee la fricativa labial sorda / $\phi /$ como fonema; como mucho, se encuentra en fonética como variante de las fricativas fuertes $/ \mathrm{s} t \int \mathrm{h}^{\mathrm{w}} \mathrm{h} /$. En cambio, otras lenguas tucanas sí poseen la labial sorda, como el tamicuyalo en el numeral 'cinco' /i?ra фi?ta/, y la

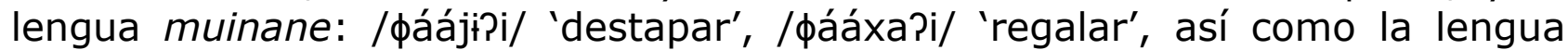
andoque, en la que aparece en posición medial principalmente y tiene función fonológica distintiva frente $a / b /, / r /, / s / y / h /$. También la tiene la lengua

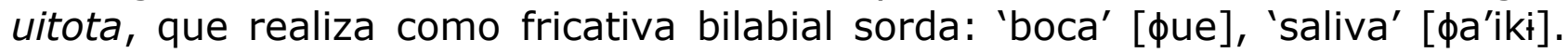
Sin embargo, carecen de ella la lengua bora y la miraña.

\subsection{Grafemas de sonidos fricativos: el dígrafo <sz>}

El dígrafo <sz> que aparece en el Vocabulario es una de las unidades grafémicas más alejadas del inventario grafémico español:

(9)

Ms-RAH

Szia - el huevo

Sziacoróbue - Cascara de huebo

Szanquin - Mascar
$M s-B N C$

Ceñósziaque - Yema de huevo

Kẽenszia - la liendre

En el Vocabulario, los vocablos que tienen el dígrafo <sz> como inicial se alfabetizan bajo la letra " $S$ " más la vocal correspondiente: <a> por ej. en Szanquin - 'mascar' e <i> por ej. en Szia - 'el huevo'. La colocación de estas entradas en el Vocabulario encuentra explicación desde la reconstrucción fonológica de las consonantes de la serie coronal ${ }^{*} s,{ }^{*} c$, ${ }^{*} t j$ y ${ }^{*} j$, que, según Chacon (2014: 301), conduce en las lenguas tucanas a un complejo sistema de realizaciones alofónicas de las sibilantes, las alveopalatales y las palatales, resultado de la fusión entre ellas. Las posiciones más afectadas por el cambio son la semivocal $*_{j}$ inicial y la alveolar ${ }^{*} s$ inicial y los cambios más relevantes históricamente en la lengua siona son la fusión de ${ }^{*} t j$ con ${ }^{*} s$ y el paso de la ejectiva $* t j^{\prime}$ a la correspondiente $s^{\prime}$, mientras que en koreguaje $* t j^{\prime}$ se fusionó con $* j$. Esa divergencia en el resultado de las fusiones es importante a la hora de dilucidar las consonantes que se pueden "reconstruir" detrás del dígrafo $<\mathrm{sZ}>$.

En la reconstrucción de consonantes del prototucano, nos interesa el destino de la alveopalatal africada *ts y la variante eyectiva correspondiente *ts' (Ladefoged 2001: 131-133), las cuales conforman la fase más extendida de fusión junto con la africada palatal *tf. Esto se debe a que, en lingüística histórica, desde testimonios históricos y no reconstructivos, se corrobora que el dígrafo <sz> se relaciona fonológicamente con una consonante africada alveopalatal, tanto sorda como sonora. Y es en la actualidad de la lengua koreguaje en la que en este vocablo 'huevos' /t fiá/ (Rodríguez González 2000: 201) tiene una consonante africada palatal, lo que se correspondería, trasladando al pasado, con la africada alveopalatal del dígrafo <sz>.

En cambio, en la lengua siona actual el ejemplo Szia - el huevo se corresponde con fricativa sonora (o suave): /'zia-bi/ [ziabi] 'el huevo' (con el sufijo nominalizador $-b \dot{t}$ 'objeto de forma redondeada 0 esférica, 0 que 
manifiesta una protuberancia'), según Wheeler (2000: 184-185), o con la fricativa coronal: /s'iá-t'abi-t't'/ [s'iára?phi' ri] (Chacon 2014: 298).

La conclusión es que, en este caso, la grafémica nos conduce a situar como la más plausible la pronunciación africada del koreguaje o de aquellas otras lenguas amazónicas que conservan la serie africada hasta hoy ${ }^{12}$.

\subsection{Grafemas oclusivos}

Desde el método reconstructivo se propugna que en las lenguas del grupo tucano occidental la consonante inicial del grupo de consonantes velares $\mathrm{k}, \mathrm{g}$ o kw sería *k (Skilton 2013: 39). En uno y otro manuscrito las variantes grafémicas $\langle\mathrm{c}\rangle$ y $\langle\mathrm{k}\rangle$ alternan en posición inicial y medial en muchos casos sin dejar traslucir ningún rastro de correspondencia en fonología con otra pronunciación que no sea la velar fuerte $k$, si bien preferentemente se produce en el contexto de grafemas vocálicos $\langle a\rangle$, $<u>$ (Wheeler 2000: 186).

Cancokan - Corteza de que se visten,

Cankocan - cortesa de un árbol de que se visten o Damajahua que se llama Damajagua

Por el resultado que se observa en la actualidad, no es descartable que, bajo variantes grafémicas como $\langle\mathrm{c}\rangle \mathrm{y}\langle\mathrm{k}\rangle$, se pudieran encerrar distintos cambios fonológicos. En la lengua siona actual, las variantes grafémicas $\langle c\rangle$ y $<k>$ de distintas entradas léxicas del Vocabulario, implican en fonología un proceso de suavización de la tensión articulatoria hacia la velar suave $g$.

(11)

Ms-RAH

Cohy - volver, ó volverse

Kante - Caña braba

Kéna - El fierro, ú otro metal
MS-BNC

Cohi - volver o volverse

/ga'tija/ 'el río de la cañabrava'

o 'Putumayo' (con el morfema -ja 'río o quebrada')

(Wheeler 2000: 186)

/gi'na/ 'metal, vidrio, plástico' (Wheeler 2000: 186).
Siona actual

/go'pi/'regresar'

(Wheeler 2000: 191)

El cambio se produciría en posición faringea o glotal inicial, pese a que el método reconstructivo vincula el paso $* k>g$ sobre todo a la posición faringea o glotal medial (Chacon 2014: 287).

Otro cambio que puede suponerse detrás de la variante gráfica $\langle c\rangle$ es la aspiración de la oclusiva fuerte velar, $k^{h}$. En esta ocasión participarían del cambio $* k>k^{h}$, entre otras lenguas tucanas, la siona, la koreguaje y la wanano (Chacon 2014: 287).

\footnotetext{
12 El muinane tiene africadas alveopalatales sorda / $\mathrm{t} /$ y sonora / $\mathrm{d} 3 / \mathrm{y}$ fricativa alveopalatal sorda /S/ (Walton et al. 2000: 256); el bora, que tiene el fonema / $\mathrm{t} /$ se opone a /j/, pero no tiene el fonema alveopalatal sonoro (Escobar 2000: 246); el ocaina, con un sistema consonántico muy completo en el orden alveopalatal: dos oclusivas, dos fricativas, dos africadas ( $\check{c} / \mathrm{j}^{\nu}=\mathrm{t} / / \mathrm{d} 3$ ) (Patiño Roselli 2000: 239), y el uitoto: /c $\mathrm{t} /$, con oclusivas palatales,

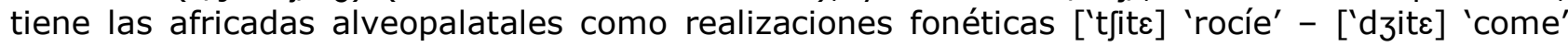
(Petersen de Piñeros y Patiño Rosselli 2000: 221).
} 


\section{(12)}

Ms-RAH / MS-BNC

Coó - La uña / Coô - la vña

Coneguay - Un pájaro
Siona actual

/'kowi/ ['k $\left.{ }^{\mathrm{h}} \mathrm{o}^{\prime} w i\right]$ 'uña' en siona (Wheeler 2000: 182)

/ko'ne/ [k'o'nææ] 'pájaro carpintero' en siona (Wheeler 2000: 183)

La alternancia en los dos manuscritos en el uso de los dígrafos <qu> y $<$ gu $>$ ante <e> en entradas léxicas idénticas obedece a fenómenos fonológicos subyacentes. El Ms-RAH se sirve del grafema oclusivo sordo (fuerte), mientras que el Ms-BNC prefiere el grafema oclusivo sonoro (suave).

(13)

\section{Ms-RAH}

Buhuque - La madriguera

Ciayaqueque - Vaca marina

\author{
Ms-BNC \\ Buhuguè - Madriguera de Guatusas \\ Ciaya gueque - Vaca marina
}

El Ms-BNC se aproxima así a la tendencia actual en las lenguas tucanas occidentales $y$, en particular, en la lengua siona, en la que se observa la confluencia fonológica entre $k^{w}$ y $g^{w}$ a favor de una velar fonológicamente suave (sonora) e incluso con glotalización: /gu'Pahi/ [ku'Pahĩ] (Wheeler 2000: 196). Además, el análisis de reconstrucción propone la existencia del paso $* k$ $>t$ en posición inicial, ante vocales $e, i$, con la apertura posterior en $a$ en algunas lenguas del grupo tucano, como el wanano, cerca del Vaupés (Waltz 2002, cit. por Chacon 2014: 287).

Esta situación parece reflejarse en aquellas entradas léxicas del Vocabulario que tienen un dígrafo <ch > en posición inicial y medial de palabra y que fonológicamente suele conrresponderse con la africada palatal sorda (o fuerte) $t$ :

\section{(14)}

Ms-RAH

Chaó - Tirar con honda

Chacú - Tirar con arco y flecha

Chacoyari - Flecha, saeta

Chaomea - La honda

Ms-BNC

Chaõ - tirar con onda

Chaco - tirar con flecha

Chaco Jari - la fecha o saeta

Chaomeà - la onda
Ms-RAH/Ms-BNC

Jochá - Estar ágrio

Jochaseé - Agrio

\subsection{Grafemas de la semivocal labial}

La semivocal labial $/ w /$, que está presente en prácticamente todas las lenguas tucanas, experimenta históricamente diferentes cambios motivados por el contexto fonético en el que se encuentra. A través de la grafémica se descubre que la secuencia grafémica <hu> posee en siona actual el valor fonológico de una semivocal labial.

(15)

Ms-RAH / Ms-BNC

Huiyape - Manteca, ó azeite en común

Huytocen - El anzuelo
SIONA ACTUAL

[wi?jabe] 'la manteca' (Wheeler 2000: 185)

[wi'tosẽ'?ẽ] 'el anzuelo' (Wheeler 2000: 186) (sufijo nominalizador -sẽ'?ẽ 'algo que sirve para enganchar o agarrar') 
La secuencia grafémica $<$ hu $>$ ante vocal $<$ a $>$ aparece en alternancia con $<$ gu $>$, tal como se recoge en una entrada léxica: (Ms-RAH) Huacarà - ave / $(M s-B N C)$ Guacharaca. El contexto grafémico $<$ hu $>/<$ gu $>$ es descrito, desde el método reconstructivo, en conexión con cambios en el nivel fonológico que afectan a la semivocal labial $* w$, o mejor dicho, a "la coarticulación de la protosecuencia *wu" (Chacon 2014: 308). Un ejemplo significativo es la distinta solución adoptada en cada manuscrito en la secuencia grafémica <hu> y <gu> en el sufijo sustantivador -huatí / -guatí, un sufijo que alterna, en cuanto a la semasia, con-pempe/-penpe.

Ms-RAH

Comehuati, ó Comepempe - La cuchara

Chacohuati - El arco con que se tira
Ms-BNC

Comeguati, l. Comepenpe - la cuchara

Chacò guatí $=$ \{tachado el que sabe tirar $\}$ Arco con que se tira

\subsection{Grafemas vinculados a sonidos glotales}

Uno de los aspectos de mayor complejidad en el Vocabulario es el nivel de coocurrencia y el carácter multifuncional de los grafemas $\langle i\rangle,\langle y\rangle,\langle j\rangle$. Podría pensarse que se trata de distribución de meras variantes gráficas, según la cual, por ejemplo, el Ms-RAH sitúa $<y>$ en los mismos vocablos en los que el Ms-BCN opta por las variedades grafémicas $<\mathrm{i}>0<\mathrm{j}>$ : $(M s-R A H)$ Sayéséyo - apedrear / (Ms-BNC) Saiesejo. Pero no es así, sino que en la lengua siona las distintas variantes gráficas tienen realizaciones fonéticas distintas. Así, por ejemplo, la variante grafémica $<j>$ medial contiene el valor fonológico de un sonido glotal fricativo /h/, que desde la reconstrucción del consonantismo prototucano se describe como frecuente en esa posición y asociado con picos tonales altos y fuerzas acentuales.

(17)

Ms-RAH

Ceñojañé - Color amarillo

$M s-R A H / M s-B C N$

Yéja - La tierra, el suelo, ó cosa baja

\author{
Ms-BCN \\ Cenõjanẽ - Color amarillo
}

SIONA ACTUAL

/s T̛'jõ'hẽihĩ/ [s '̂'nõ-'hǣî-hĩ]

'es amarillo' (Wheeler 2000: 184)

El carácter glotal que encierra la consonante detrás de $\langle\mathrm{j}\rangle$ queda confirmado en otro ejemplo, que introduce un grafema nuevo en uno de los dos manuscritos: donde el Ms-BNC utiliza $<j>$ el Ms-RAH había escrito un grafema $\langle x\rangle$, ambos representativos, en la ortografía española del siglo XVIII, de una consonante bien fricativa velar sorda $[\mathrm{X}]$ o bien de un sonido laríngeo [h] en pronunciación dialectal.

El grafema $\langle y>$ compagina diferentes valores. En Ms-RAH aparece el grafema $<y>$ en contextos en los que en Ms-BNC se encuentra el grafema de la aspiración $<\mathrm{h}>$. 
(19)

Ms-RAH

Ayiñe - Blando
Ms-BNC

Ahinẽ - blando

En primera posición de una entrada léxica, como Yéja /ji'ha/, marca una semivocal palatal $/ \mathrm{j} /$; en cambio, en posición de secuencia vocálica es grafema de la vocal anterior /i/.

En contexto de dos vocales, puede desarrollarse un proceso de glotalización. En el Vocabulario se ejemplifica con un sufijo <-may>, hoy día fonológicamente /-'ma'?i/, que precede a la flexión y con el que se señala la negación en siona (Wheeler 2000: 184).

(20)

MS-RAH

Say - Irse, ó hazer que se vaya

Saymay - No irse

/'sai-'ma'?i/

Achá - Oir

Acha-may - No oyr

/Pa'tJahi-'ma'?i/

\author{
Ms-BNC \\ Say - irse / Sai - irse \\ Acha - oir
}

En otras ocasiones, en la secuencia de dos vocales, la segunda de ellas, vocal alta anterior [i], graficada $<\mathrm{y}>$ en $M s-R A H$ e $<\mathrm{i}>$ en $M s-B N C$, se marca ya la aspiración recurriendo al grafema consonántico aspirado $\langle\mathrm{h}\rangle$. Es lo que sucede con la entrada léxica hay - comer / ahí - comer, que en siona actual tiene la pronunciación aspirada /ehi/ [ehĩ] 'comer' (Wheeler 2000: 182). En posición medial intervocálica se puede colegir que el correlato fonológico del grafema $<\mathrm{h}>$ es la fricativa laríngea o glotal, tal como puede verse en la forma del prototucano *gohe 'agujero' (Skilton 2013: 21).

Una nueva función del grafema $<y>$ es la que ofrece el Ms-RAH en el mismo contexto en el que el $M s-B N C$ sitúa un grafema consonántico nasal $<\tilde{n}>$.

Este hecho conecta con la descripción en el prototucano del sonido [n] como alófono de la semivocal palatal $* j$ (Chacon 2014: 308).

(21)

Ms-RAH

Kiyo - Quitar, apartarse, ó apartar á otro
Ms-BNC

Kiño - quitar, aportarse, ô apartar a ôtro

\subsection{Grafemas oclusivos geminados y procesos de laringalización}

En la descripción de la fonología del prototucano se afirma la existencia de oclusivas geminadas dentales $* t t$ y velares $* k k$ en posición medial, que conducen a un radical (C)VCCV y que tiene distinto desarrollo según las áreas geográficas y no tanto según los diferentes subgrupos (Chacon 2014: 288). En el caso del Vocabulario, es precisamente el Ms-BNC el que presenta la variante grafémica de la oclusiva geminada explícita $<\mathrm{tt}>$ en posición medial, precedido de un grafema <-c> en posición implosiva y también un grafema oclusivo geminado $<\mathrm{tt}>$ precedido de un grafema nasal $<\mathrm{n}>$. 
(22)

Ms-RAH

Octa, ó Oy - Llorar

Octé - Sembrar maíz

Roctá - Acordarse, ó pensar

Rintó - Picar como avispa

\author{
Ms-BCN \\ Octta. I. oý - Ilorar \\ Octtē - sembrar mais \\ Roctta - pensar, ò acordar[se] \\ Rintto - picar como abispa
}

No obstante, los caracteres grafémicos de esos mismos vocablos en el Ms$R A H$, con diferentes variantes grafémicas, la oclusiva sorda $<-c>$, la oclusiva suave <-g> y el grafema consonántico laríngeo <-h> en posición implosiva representan el proceso de lenición de la oclusiva geminada en una preaspiración ([h], [h]) o en una glotalización [?] (Ladefoged y Maddieson 1996: 325).

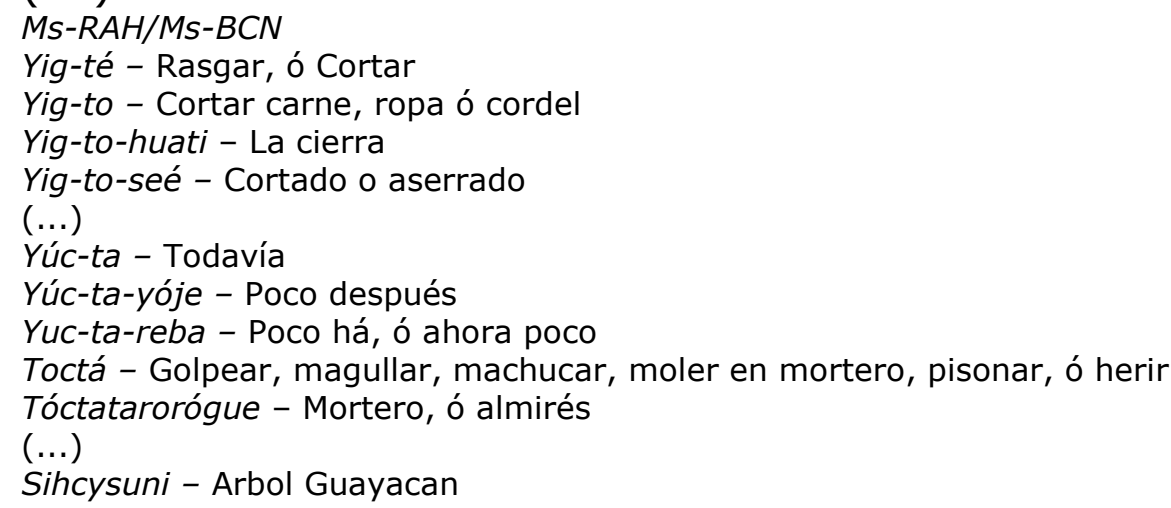

\section{A modo de conclusión}

Las fronteras entre los distintos grupos lingüísticos son extremadamente difusas, tal como se observa hoy en día en los cruces e intersecciones de símbolos representativos de la distribución de las distintas familias amazónicas occidentales (Chacon 2014: 177). No extraña, por lo tanto, que Tovar (1961: 212) indicara, con referencia al texto del Ms-RAH, que el Vocabulario, pertenecía al grupo lingüístico tucano, sin mayor precisión, y más adelante, como mucho, Tovar y Larrucea de Tovar (1984: 230) lograran una mayor precisión al situarlo en el tucano occidental. Se imponía un análisis sistemático y exhaustivo del contenido lingüístico del texto, así como resultaba imprescindible por poner en claro aquellas cuestiones puramente filológicas y de trasmisión que estaban pendientes hasta este momento.

En el estudio, se ha visto la enorme utilidad de la propuesta de Landaburu (2004-2005: 4-5) de tomar en cuenta los resultados aportados por los investigadores que entran en contacto directo con las distintas familias lingüísticas. De ahí que este trabajo esté en deuda con la descripción lingüística de Wheeler (1987a, 1987b, 2000: 181-198) y Wheeler y Wheeler (1992) para la lengua siona, y de Rodríguez González (2000: 199-213) para la lengua koreguaje. Para el análisis grafofonológico y el estudio de las variantes grafofonéticas, resultan aún del todo imprescindibles, sin dejar de lado el resto de descripciones de otras familias lingüísticas de las regiones amazónicas meridional y septentrional llevadas a cabo hasta la fecha. 
El nivel grafémico y los correlatos fonéticos y fonológicos de los grafemas y grafías han dado resultados confiables. Observamos algunos fenómenos que vinculan el texto con la rama del tucano oriental y de otras familias lingüísticas de la zona amazónica septentrional (uitoto, muinane-bora, piaparaná, etc.). Resulta especialmente interesante el grafema $<f>$ presente en palabras no pertenecientes al tucano occidental, pues ni el siona ni el koreguaje poseen el fonema fricativa labial sorda $/ \phi /$, mientras que otras lenguas tucanas sí poseen la labial sorda, como el tamicuyalo, la lengua andoque y la lengua uitota, aunque carecen de ella la lengua bora y la miraña.

Estos rasgos son minoría en comparación con los rasgos que relacionan la fonología del vocabulario con el tucano occidental, en concreto con las lenguas siona y koreguaje, ambas con aportaciones decisivas en este nivel lingüístico de análisis. Ha quedado patente, también, que cada uno de los dos manuscritos, a los que respectivamente hemos calificado como Ms-RAH (quitense) y Ms-BNC (payanés), guarda una gran proximidad con cada una de las dos lenguas más importantes del subgrupo: las tendencias grafofonémicas del Ms-RAH lo aproximan a la lengua koreguaje, mientras que las del Ms-BNC se ciñen de manera más ajustada a la lengua siona.

\section{Referencias bibliográficas}

Barnes, Janet. 1999. Tucano, en Robert M. W. Dixon y Alexandra Y. Aikhenvald (eds.), The Amazonian languages, Cambridge, Cambridge University Press: 207-26.

Carrera de la Red, Micaela. 2015. Variantes grafémicas y correlatos fonológicos en la lengua tucana de un Vocabulario de la Amazonía ecuato-colombiana (siglo XVIII), Comunicación presentada en el XLIV Simposio de la Sociedad Española de Lingüística, Madrid, CCHS-CSIC, 27-30 enero 2015.

Carrera de la red, Micaela. 2016. Mecanismos "clasificadores" en el tucano occidental del Vocabulario de la lengua general de los indios del Putumayo y Caquetá (siglo XIX), Comunicación presentada en Congreso sobre Español de América: historia y contactos, Pisac, Cuzco, Perú, 11-15 julio, 2016.

Carrera de la Red, Micaela y Francisco José Zamora Salamanca. 2014. Un intento de configuración de lengua general en el sur de Colombia: el Vocabulario de la lengua general de los indios del Putumayo y Caquetá, Comunicación presentada en el VIII Congreso Internacional de Lingüística Misionera, Lima, 25-28 marzo, 2014.

Chacon, Thiago. 2012. The phonology and morphology of Kubeo: The documentation, theory and description of an Amazonian language, Tesis Doctoral. University of Hawaii, Manoa, Honolulu [en línea] Disponible en https://scholarspace.manoa.hawaii.edu/bitstream/10125/101344/1/Chaco n_Thiago_r.pdf

Chacon, Thiago. 2014. A revised proposal of Proto-Tukanoan consonants and Tukanoan family classification, International Journal of American Linguistics, 80, 3: 275-322.

Cook, Dorothy M. y Linda L. Criswell. 1993. El idioma koreguaje (tucano occidental), Bogotá, Instituto Lingüístico de Verano. 
Elías Ortiz, Sergio. 1942. Bocabulario de la lengua que usan los indios de estas misiones, Ceona, Revista de Historia, 2: 137-199.

Epps, Patience y Andrés Salanova. 2013. The Languages of Amazonia. Tipití: Journal of the Society for the Anthropology of Lowland South America, 11, 1: $1-28$.

Escobar, Rosa Alicia. 2000. Aproximación a la fonología del bora, en María Stella González de Pérez y María Luisa Rodríguez de Montes (coords.), Lenguas indígenas de Colombia: una visión perspectiva, Bogotá, Instituto Caro y Cuervo, 243-252.

Fabre, Alain. 1998. Manual de las lenguas indígenas sudamericanas, Munich, Lincom-Europa.

Gilij, Filippo Salvadore. 1780. Saggio di Storia americana, o sia, Storia naturale, civile e sacra de regni e delle provincie Spagnuole di Terra-ferma nell'America meridionale, Roma, Vaticano nella Sapienza.

Gomez-Imbert, Elsa. 2000. Introducción al estudio de las lenguas del Piraparaná (Vaupés). Lenguas indígenas de Colombia: Una visión descriptiva, en María Stella González de Pérez y María Luisa Rodríguez de Montes (coords.), Lenguas indígenas de Colombia: una visión perspectiva, Bogotá, Instituto Caro y Cuervo: 321-356.

Gomez-Imbert, Elsa. 2003. Voir et entendre comme sources de connaissance grammaticalement explicites, en C. Vandeloise (ed.), Langues et cognition, Paris, Hermès Science Publications: 117-133.

Gomez-Imbert, Elsa. 2004. Fonología de dos idiomas tukano del Piraparaná: barasana y tatuyo, Amerindia, 29: 43-80.

Gomez-Imbert, Elsa. 2007. Tatuyo and Other Tukanoan Class Systems, en Leo W. Wetzels (ed.), Language Endangerment and Endangered Languages: Linguistic and Anhropological Studies with Special Emphasis on the Languages and Cultures of the Andean-Amazonian Border Area, Leiden, Research School of Asian, African and Amerindian Studies (CNWS): 401-428.

Gomez-Imbert, Elsa. 2011. La famille Tukano, en E. Bonvini, J. Busuttil y A. Peyraube (eds.), Dictionnaire des langues, Paris, Presses Univ. de France.

Gómez, Diego F. y Jennifer Torres (eds.). 2012-2013. Vocabulario ceona, doctrina cristiana, interrogatorio para confesión, etc. Manuscrito II/2916 Biblioteca del Palacio Real de Madrid, Bogotá, Instituto Colombiano de Antropología e Historia. [en línea] Disponible en: http://coleccionmutis.cubun.org/Manuscrito_2916_BPRM_todo

González Mora, Felipe. 2004. Reducciones y haciendas jesuíticas en Casanare, Meta y Orinoco ss. XVII-XVIII. Arquitectura y urbanismo en la frontera oriental del Nuevo Reino de Granada, Bogotá, Editorial Pontificia Universidad Javeriana.

Jackson, Jean. 1974. Language Identity of the Colombian Vaupés Indians, en R. Bauman y J. Sherzer (eds.), Explorations in the Ethnography of Speaking, New York, Cambridge University Press: 50-64.

Jackson, Jean. 1983. The Fish People. Linguistic Exogamy and Tukanoan Identity in Northwest Amazon, Cambridge, Cambridge University Press. 
Jackson, Jean. 2001. Treinta años estudiando el Vaupés: Lecciones y reflexiones, en Carlos E. Franky y Carlos G. Zárate (ed.), IMANI MUNDO: Estudios en la Amazonia colombiana, 18: 11-51.

Jiménez de la Espada, Marcos. 1898-1899. Vocabulario de la lengua general de los indios del Putumayo y Caquetá, Revista de Archivos, Bibliotecas y Museos, T. II, 5: 200-216; 6: 258-263; 7: 427-432; 8: 527-529; 12: 575-577; T. III, 3-4: 187-791; 6: 358-362; 8: 518-520.

Jiménez de la Espada, Marcos. 1904. Vocabulario de la lengua general de los indios del Putumayo y Caquetá, Madrid, Tipografía de la Revista de Archivos, Bibliotecas y Museos.

Johnson, Orville E. y Catherine Peeke. 1962. Phonemic Units in the Secoya Word, en Catherine Peeke y Benjamin Elson (eds.), Studies in Ecuadorian Indian Languages, México D.F., Instituto Lingüístico de Verano: 78-95.

Johnson, Orville E. y Stephen H. Levinsohn. 1990. Gramática Secoya. Quito, Instituto Lingüístico de Verano.

Ladefoged, Peter. 2001. Vowels and Consonants. An Introduction to the Sounds of Laguages, Oxford/Malden, Blackwell.

Ladefoged, Peter y Ian Maddieson. 1996 The Sounds of the World's Languages, Oxford/Malden, Blackwell.

Landaburu, Jon (ed.). 1996. Documentos sobre lenguas aborígenes de Colombia del archivo de Paul Rivet, Bogotá, Ediciones Uniandes-CCELAColciencias.

Landaburu, Jon. 2004-2005. Las lenguas indígenas de Colombia: presentación y estado del arte, Amerindia, 29/30: 3-22.

Larrucea de Tovar, Consuelo. 1986. José Celestino Mutis (1732-1808) and the report on American languages ordered by Charles III of Spain for Catherine the Great of Russia, en Antonio Quilis Morales y Hans Josef Niederehe (eds.), The History of Linguistics in Spain, Amsterdam, John Benjamins: 213-231.

Loukotka, Čestmír. 1935. Classification of South American Indian Languages, Los Angeles, UCLA Latin American Center.

Mantilla, Luis Carlos. 1986. Cali y su convento de San Francisco: documentos inéditos: homenaje de los franciscanos de Colombia a Cali en sus 450 años, Bogotá, Editorial Kelly.

Moreno Gallego, Valentín. 2009. La Real biblioteca y sus fondos americanistas: guía de fuentes, en Lope de Barrientos Seminario de Cultura, 2: 105-140.

Muller de Young, Carolyn, Dorothy Cook y Frances Gralow. 1984. Fonología del coreguaje, Sistemas fonológicos de idiomas colombianos. Vol. V. Lomalinda, Editorial Townsend: 63-79.

Ortega Ricaurte, Carmen. 1997. Los manuscritos sobre lenguas indígenas en la Biblioteca General, en Senderos, 8, 31/32: 1142-1148.

Patiño Roselli, Carlos. 2000. Reseña bibliográfica del ocaina, en María Estella González Pérez y María Luisa Rodríguez de Montes (coords.), Las lenguas indígenas de Colombia. Una visión descriptiva, Bogotá, Instituto Caro y Cuervo:139-141.

Petersen de Piñeros, Gabriele y Carlos Patiño Rosselli. 2000. El idioma uitoto, en María Stella González de Pérez y María Luisa Rodríguez de Montes 
(coords.), Lenguas indígenas de Colombia: una visión perspectiva, Bogotá, Instituto Caro y Cuervo: 219-238.

Rodríguez González, Sandra Patricia. 2000. Estudios sobre la lengua koreguaje, en María Stella González de Pérez y María Luisa Rodríguez de Montes (coords.), Lenguas indígenas de Colombia: una visión perspectiva, Bogotá, Instituto Caro y Cuervo: 199-214.

Silva, Wilson D. L. 2012. A Descriptive Grammar of Desano,Tesis doctoral, University of Utha, Salt Lake City (inédito).

Skilton, Amalia. 2013. A new proposal of Western Tukanoan consonants and internal classification, Yale, University of Yale Press.

Sorensen, Arthur P. Jr. 1972. Multilingualism in the Northwest Amazon, en J. B. Pride, y Janet Holmes (eds.), Sociolinguistics. Selected Readings, Middlesex, Penguin Books: 78-93.

Stenzel, Kristine. 2007. Glottalization and Other Suprasegmental Features in Wanano. International Journal of American linguistics, 73, 3: 331-366.

Tovar, Antonio. 1961. Catálogo de las lenguas de América del Sur, Buenos Aires, Editorial Sudamericana.

Tovar, Antonio y Consuelo Larrucea de Tovar. 1984. Catálogo de las lenguas de América del Sur, Madrid, Gredos.

Vallejos, Rosa. 2013. El secoya del Putumayo: aportes fonológicos para la reconstrucción del Proto-Tucano Occidental, Liames, 13: 67-100.

Walton, James, Grace Hensarling y Michael R. Maxwell. 2000. El muinane, en María Stella González de Pérez y María Luisa Rodríguez de Montes (coords.), Lenguas indígenas de Colombia: una visión perspectiva, Bogotá, Instituto Caro y Cuervo: 255-274.

Waltz, Nathan E. 2002. Innovations in Wanano (Eastern Tocanoan) when compared to Piratapuyo, International Journal of American Linguistics, 68: 157-215.

Wheeler, Alva. 1987a. El pueblo siona del río Putumayo, Colombia. Vol. 1: Etnología, gramática, textos, Bogotá, Instituto Lingüístico de Verano.

Wheeler, Alva. 1987b. El pueblo siona del río Putumayo, Colombia. Vol. 2: Diccionario, Bogotá, Instituto Lingüístico de Verano.

Wheeler, Alva. 1992. Comparaciones lingüísticas en el grupo tucano occidental, en Alva Wheeler y Margaret Wheeler (eds.), Estudios Comparativos: Proto Tucano. Janet Barnes, Bogotá: 17-53.

Wheeler, Alva. 2000. La lengua siona, en María Estella González Pérez y María Luisa Rodríguez de Montes (coords.), Las lenguas indígenas de Colombia. Una visión descriptiva, Bogotá, Instituto Caro y Cuervo: 181-198.

Wheeler, Alva y Margaret Wheeler (eds.). 1992. Estudios Comparativos: Proto Tucano, Bogotá, Janet Barnes.

Zawadzky, Alfonso. 1947. Viajes misioneros del R.P.Fr. Fernando de Jesús Larrea, franciscano, 1700-1773, Cali, Impresora Bolivariana.

NOTA:

La autora de este artículo es la única responsable por su contenido y redacción. 